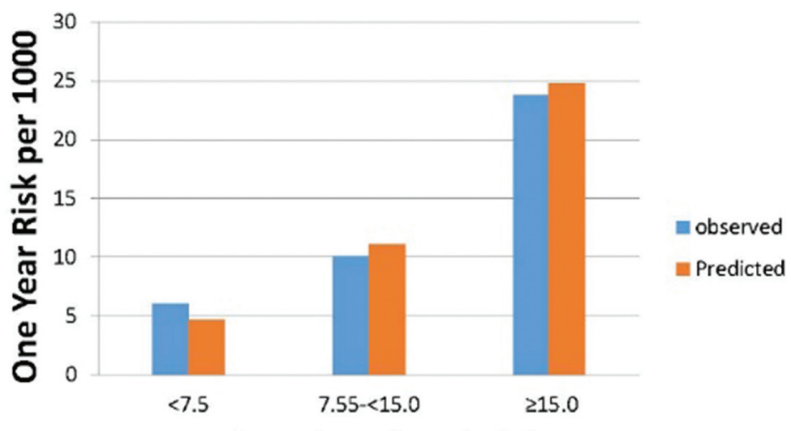

Group of Predicted probabilty, per 1000

Abstract SAT0699 - Figure 1. Observed vs. Predicted One-Year CVD Risk per 1000 person-years in RA Patients, using only Age, Sex, Race, and MBDA score (Model 3)

Conclusions: Preliminary results from this analysis suggest that a simple algorithm consisting only of age, sex and race plus a multi-biomarker score can provide an accurate method to predict short term CVD risk in RA. Further validation with more extended time frames should improve the utility of this approach.

Acknowledgements: This work was supported by the Patient Centred Outcomes Research Institute (PCORI) and Myriad Genetics.

Disclosure of Interest: F. Xie: None declared, L. Chen: None declared, H. Yun Grant/research support from: BMS, J. Curtis Grant/research support from: AbbVie, Amgen, BMS, Corrona, Janssen, Lilly, Myriad, Pfizer, Roche/Genentech, UCB, Consultant for: AbbVie, Amgen, BMS, Corrona, Janssen, Lilly, Myriad, Pfizer, Roche/Genentech, UCB

DOI: 10.1136/annrheumdis-2018-eular.5726

\section{SAT0700 \\ THE RELATIONSHIP BETWEEN MUSCULOSKELTAL PAIN, INFLAMMATION AND DEPRESSION IN MEN}

J. McBeth ${ }^{1}$, M.J. Cook ${ }^{1}$, G. Bartfai ${ }^{2}$, F. Casanueva ${ }^{3}$, M. Maggi ${ }^{4}$, A. Giwercman ${ }^{5}$ D. Vanderschueren ${ }^{6}$, J. Slowikowska-Hilczer ${ }^{7}$, M. Punab ${ }^{8}$, T.W. O'Neill ${ }^{1,9,10}{ }^{1}$ The University of Manchester, Manchester, UK; ${ }^{2}$ Medical University, Szeged, Hungary; ${ }^{3}$ Santiago de Compostela University, Santiago de Compostela, Spain; ${ }^{4}$ University of Florence, Florence, Italy; ${ }^{5}$ Lund University, Malmö, Sweden; ${ }^{6}$ Katholieke Universiteit Leuven, Leuven, Belgium; ${ }^{7}$ Medical University of Lodz, Lodz, Poland; ${ }^{8}$ United Laboratories of Tartu University Clinics, Tartu, Estonia; ${ }^{9}$ NIHR Manchester Biomedical Research Centre, Central Manchester University Hospitals NHS Foundation Trust, Manchester, ${ }^{10}$ Salford Royal NHS Foundation Trust, Salford, UK

Background: Up to $40 \%$ of persons with musculoskeletal (MSK) pain report depression. High sensitivity C-reactive protein (hsCRP), a sensitive marker of chronic low grade inflammation, is elevated in persons with MSK pain when compared to those who are pain free, and in those with MSK pain is positively associated with increasing pain severity. High levels of hsCRP are also associated with depression. Whether hsCRP is the mechanism linking MSK pain to depression has not been tested.

Objectives: To test the hypothesis that MSK pain predicts depression and the relationship would at least in part be explained by hsCRP.

Methods: 3309 men aged 40-79 in the multicentre European Male Ageing Study completed a postal questionnaire asking about MSK pain (classified as chronic widespread pain American College of Rheumatology 1990 criteria) (CWP), some pain that was not CWP (SP), or no pain (NP)) and depression (Beck Depression InventoryII (BDI-II), score range 0-21). At a research clinic, fasting morning blood samples were shipped to one Centre where hsCRP was measured using a solid-phase, chemiluminescent immunometric assay with a sensitivity of $0.01 \mathrm{mg} / \mathrm{dL}$. Covariates were date of birth, education level, body mass index (BMI), tobacco use, and frequency of alcohol consumption. Participants were followed up (mean 4.4 (SD 0.3 ) years) and completed the BDI-II. Linear regression tested the association between baseline pain and follow up BDI score (outcome), with adjustments for age, centre and baseline BDI. In a final model hsCRP and covariates were added and the impact on the relationship between pain and BDI-II examined. Results are expressed as $\beta$ coefficients with $95 \%$ confidence intervals $(\mathrm{Cl})$.
Results: 2404 (72.7\%) men had complete baseline and follow-up data. At baseline 1003 (41.7\%) reported NP, 1205 (50.1\%) SP, and 196 (8.2\%) CWP. Mean (SD) age was 59.3 (10.6), CRP 0.41 (0.74) mg/l, and BDI 6.6 (6.2). Mean BMI was 27.8 (10.6), 30.1\% were current smokers, 23.6\% drank alcohol 5-7 days/week and $53.9 \%$ had gone on to further education. After adjusting for age, Centre and baseline BDI-II when compared to those with NP having SP $(\beta=0.7,95 \% \mathrm{Cl}(0.3$, $1.1))$ or CWP $(1.3(0.5,2.0))$ at baseline was associated with higher BDI-II scores at follow-up. Baseline hs-CRP did not predict follow up BDI-II $(0.05(-0.2,0.3))$. The relationship between $\operatorname{SP}(0.7(0.2,1.1))$ or $\operatorname{CWP}(1.5(0.7,2.3))$ and follow up BDI-II persisted after adjustment for hs-CRP and all covariates.

Conclusions: The excess rate of depression in men with MSK pain remains to be explained. A broader assessment of inflammatory markers (e.g. TNF- $\alpha$, Interleukins, and IFN- $\gamma 1$ ) may identify mechanisms linking MSK pain with subsequent depression.

Acknowledgements: The European Make Ageing Study was funded by the Commission of the European Communities Fifth Framework Programme, grant QLK6-CT-2001-00 258.

Disclosure of Interest: None declared

DOI: 10.1136/annrheumdis-2018-eular.1971

\section{SAT0701 \\ PREGNANCY OUTCOME IS IMPROVED AND SIMILAR TO THAT OF THE GENERAL OBSTETRIC POPULATION IN PATIENTS WITH RHEUMATOID ARTHRITIS WHO FOLLOW THE "IDEAL CLINICAL PATHWAY” BEFORE AND DURING PREGNANCY}

L. Andreoli ${ }^{1}$, A. Bortoluzzi ${ }^{2}$, G. Carrara ${ }^{3}$, V. Ramoni ${ }^{4}$, M. Padovan ${ }^{2}$, M. Govoni $^{2}$, A. Tincani ${ }^{1}$ on behalf of Gender Medicine SIR, C.A. Scirè ${ }^{2}$ on behalf of RECORD SIR. ${ }^{1}$ Rheumatology, University of Brescia, Brescia; ${ }^{2}$ Department of Medical Sciences, University of Ferrara, Ferrara; ${ }^{3}$ Epidemiology Unit, Società Italiana di Reumatologia - SIR, Milano; ${ }^{4}$ Rheumatology, University of Pavia, Pavia, Italy

Background: With the therapeutic advances of the last decades yielding to stable disease remission in the majority of patients, the goal of a successful pregnancy appears to be attainable for women affected by Rheumatoid Artrhitis (RA). However, individual risk stratification and measures to minimise it (e.g. withdrawal and wash-out of teratogenic drugs) are essential for the management of pregnancy in RA. $\left({ }^{1}\right.$

Objectives: This analysis aims to evaluate the adherence to a reference clinical pathway of diagnostic, pharmacological and follow-up management in women with RA, and the influence of the adherence to this pathway on the outcome of pregnancy.

Methods: Data were extracted from the Lombardy Region's (Italy) health databases for the period between 2004-2013. Patients with RA were identified through the chronic disease certification by rheumatologist (ICD9-CM code 714.0). Among these, women between the ages of 18 and 51 were selected. Data of controls from general population were also extracted. Conception has been approximated from the date of delivery or abortion. Seven healthcare quality indicators have been constructed: 1) screening of blood chemistry tests, 2) pre-conception musculoskeletal imaging, 3) pre-pregnancy antiphospholipid antibody tests, 4) ANA test and anti-ENA (antiRo/SSA) test, 5) no exposure or wash-out from teratogenic drugs (MTX/LEF); 6) no exposure to biological drugs; 7) rheumatological follow-up in outpatient visits. These 7 indicators were then summarised in 3 pathway indicators: 1) diagnostic pathway, 2) therapeutic pathway, 3) followup. The pregnancy outcome was defined on the basis of the DRG of complicated birth or abortion. Subanalysis on abortion was also done. The relationship between quality indicators and outcome variables was analysed using logistic models crude and adjusted for age and comorbidities, and the results presented as odds ratios (OR) and $95 \%$ confidence intervals $(95 \% \mathrm{Cl})$.

Results: 443 pregnancies of patients with RA were identified, with median age of 34 (IQR 31-37), median disease duration at conception of 3.8 years (IQR 1.83$6.19), 157(35.4 \%)$ of which with unfavourable outcome pregnancy outcome, of which 115 pregnancy losses. The increase or decrease of risk for those who meet specific quality indicators and pathway indicators showed a better outcome for patients screened for autoantibodies and with no exposure or washout from MTX LEF (Table). The comparisons of those who followed the ideal pathway or not compared to the general population showed a significant increase of risk only for patients not following the ideal pathway (Table). 